Research Article

\title{
Analyzing the Solution of Chemotaxis Equations with Logistic Source Term
}

\author{
Yan Xu $\mathbb{D D}^{1}$ and Mohammed Abdella Kemal $\mathbb{D}^{2}$ \\ ${ }^{1}$ Science College, Heilongjiang Bayi Agricultural University, Daqing 163319, China \\ ${ }^{2}$ Arba Minch University, Arba Minch, Ethiopia \\ Correspondence should be addressed to Mohammed Abdella Kemal; abdella.kemal@amu.edu.et
}

Received 11 May 2021; Revised 16 June 2021; Accepted 19 June 2021; Published 28 June 2021

Academic Editor: Dr. Dilbag Singh

Copyright ( 2021 Yan Xu and Mohammed Abdella Kemal. This is an open access article distributed under the Creative Commons Attribution License, which permits unrestricted use, distribution, and reproduction in any medium, provided the original work is properly cited.

\begin{abstract}
When the parameters have the characteristics of sensitivity and specificity, while the traditional analysis method is the analysis of equations, under the influence of the characteristics of parameters, because the ability to consider the overall existence and limitations of the equation is weak, this leads to the deviation of the analysis results beyond the allowable range. With the chemotactic equations with logistic source terms, a new analysis method is proposed, on the basis of setting the optimal adjustment parameters of logistic source term, the method evaluates the well-posed behavior of the solution of the chemotactic equation system with logistic source term by quantitative analysis of the global existence and limitations of the solution. The experimental results show that the proposed analysis method, after the analysis of the overall existence and limitations of the equation, has smaller deviation in the results of the appropriate qualitative evaluation and meets the analysis requirements of the solution problem of the chemotaxis equations. It can be seen that the analysis method is more practical.
\end{abstract}

\section{Introduction}

With the rapid development of science and technology, there are more and more cases that need to deal with high-dimensional data in human work and production. For these cases, we can use the method of variable selection to solve them. Whether it is regression or classification problem, the traditional analysis method is realized by using stepwise regression combined with information criterion. The practice proves that the traditional analysis method is practical, but there are many shortcomings at the same time. It is not stable to use stepwise regression method to select variables, and when there are many alternative variables of parameters, the calculation workload is huge, resulting in large calculation error, which cannot meet the requirements of the solution. Therefore, overcoming the shortcomings of the traditional analytical methods has effectively become one of the hot spots in the study of the solution of chemotactic equations. The global existence of solutions is an important direction in the study of chemotaxis. The analysis method proposed in this paper is to analyze the solution of the system of chemotaxis equations with logistic source term and realize the accurate, systematic, and comprehensive problem analysis starting from the boundedness, global existence, and limitations [1]. This method provides an important analysis method for the future development of equation system solution and also provides a technical support for the study of equation system in other fields. In Brownian motion, a particle moves in response to the instantaneous difference in impact made by bombarding molecules on different sides of the particle. In chemotaxis, the motion of a cell is influenced by the molecules of the critical substrate through a chemical interaction rather than by the direct impact characteristic of Brownian motion [2]. In order to get advantages of advanced technologies and robotics, citizens have to take a move in the face of automation farming. Certain businesses take this step in provision of agricultural machinery for lease as well as for custom hiring [3]. The present-day FEM stands on three legs: mathematical models, matrix formulation of the discrete 
equation, and computing tools to do the numerical work. The third is the one that has undergone the most dramatic changes $[4,5]$. Logistic regression analysis is a variant of traditional regression analysis for binary dependent variables which does not suffer from the weaknesses of the traditional approach to such data but which nevertheless shares many of its powerful features such as the ability to study quantitative as well as categorical independent variables and to include in the model interactions among the independent variables $[6,7]$.

\section{Analytical Method for the Solution of Chemotaxis Equations with Logistic Source Term}

2.1. Setting the Optimal Adjustment Parameters of Logistic Source Term. In the linear regression model, the essence of the method is to add penalty to the sum of squared residuals, estimate parameters, and compress coefficients at the same time and compress some coefficients to 0 to realize variable selection. The response variables in linear regression model are continuous variables, but the response variables in some regression models are not continuous variables but classified variables. At this time, the linear regression model can no longer be used. We use Lasso-logistic model to solve the problem of variable selection when response variables are classified variables, so as to determine the optimal adjustment parameters of the logistic source term.

It is known that logistic source term regression is a kind of generalized linear regression model, which is used to classify $0 / 1$ problem; that is to say, the prediction result belongs to "0" or " 1 " classification problem. Suppose there is a continuous explanatory variable $Y^{\prime}$ in theory, which represents the possibility of the event, and the value range is $W$. When the value of the variable crosses a critical point $j$, for example, when $j=0$, the event occurs. So, there are the following: when $Y^{\prime}>0, Y=1$, and in other cases, $Y=0$. Here, $Y$ is the actual observed response variable, $Y=1$ means the event occurred, and $Y=0$ means the event did not occur. Suppose that there is a linear relationship between $Y^{\prime}$ and the explanatory variable $X$, namely,

$$
Y^{\prime}=\alpha_{0}+\alpha_{1} X+\gamma
$$

In the above relationship, $\alpha_{0}$ indicates no event; $\alpha_{1}$ indicates an event has occurred; $y$ indicates an error term. Then, the probability equation of the event can be described by the following equation:

$$
\begin{aligned}
P(Y & =1 \mid X)=P\left(Y^{\prime}>0\right)=P\left[\left(\alpha_{0}+\alpha_{1} X+\gamma\right)>0\right] \\
& =P\left[\gamma>\left(-\alpha_{0}-\alpha_{1} X\right)\right] .
\end{aligned}
$$

Generally, it is assumed that the error term $y$ in (2) has logistic distribution or standard normal distribution. Since both the logistic distribution and standard normal distribution are symmetrical, we can get the following results:

$$
P\left[\gamma>\left(-\alpha_{0}-\alpha_{1} X\right)\right]=P\left[\gamma \leq \alpha_{0}+\alpha_{1} X\right]=K\left(\alpha_{0}+\alpha_{1} X\right) .
$$

According to the above equation, $\mathrm{K}\left(\alpha_{0}+\alpha_{1} X\right)$ is the distribution function of error $\gamma$, and the form of distribution function $\mathrm{K}\left(\alpha_{0}+\alpha_{1} X\right)$ depends on the hypothetical distribution of $\gamma$ [8]. When $\gamma$ is a logistic distribution,

$$
P(Y=1 \mid X)=P\left[\gamma \leq \alpha_{0}+\alpha_{1} X\right]=\frac{1}{1+e^{-\gamma}} .
$$

After simplification and treatment, the following equation is obtained:

$$
P(Y=1 \mid X)=\frac{1}{1+e^{-\left(\alpha_{0}+\alpha_{1} X\right)}},
$$

where $e$ represents a natural number. Assuming the conditional probability $P(Y=1 \mid X)=P^{\prime}$, a regression model with logistic source term can be obtained, as shown in the following equation:

$$
P^{\prime}=\frac{1}{1+e^{-\left(\alpha_{0}+\alpha_{1} X\right)}}=\frac{e^{\alpha_{0}+\alpha_{1} X}}{1+e^{\alpha_{0}+\alpha_{1} X}} .
$$

From the above calculation, it can be seen that $P^{\prime}$ is a nonlinear function of $X$, which can be transformed into a linear function. According to (6), the conditional probability that an event will not occur is defined as the following equation:

$$
1-P^{\prime}=1-\frac{e^{\alpha_{0}+\alpha_{1} X}}{1+e^{\alpha_{0}+\alpha_{1} X}}=\frac{1}{1+e^{\alpha_{0}+\alpha_{1} X}} .
$$

Based on the above two equations, the ratio of the probability of occurrence to the probability of nonoccurrence is obtained as follows:

$$
\frac{P^{\prime}}{1-P^{\prime}}=e^{\alpha_{0}+\alpha_{1} X} \text {. }
$$

This ratio is called the occurrence ratio of events and is recorded as $O$. In statistics, $\ln \left(P^{\prime} / 1-P^{\prime}\right)$ is called logit transformation [9]. The logit transformation is carried out for the regression model of the logistic source term, as shown in Figure 1.

According to the curve trend in the figure above, the transformed result can be expressed in the following linear form:

$$
\ln \left(\frac{P^{\prime}}{1-P^{\prime}}\right)=\alpha_{0}+\alpha_{1} X
$$

where $\ln (*)$ represents the base function. When there are $m$ explanatory variables in the above equation, the regression model of the corresponding logistic source term can be described by the following equation:

$$
\ln \left(\frac{P^{\prime}}{1-P^{\prime}}\right)=\alpha_{0}+\alpha_{1} X_{1}+\alpha_{2} X_{2}+\cdots+\alpha_{m} X_{m},
$$

where $P^{\prime}=P\left(Y=1 \mid X_{1}, X_{2}, \ldots, X_{k}\right)$ is the conditional probability of $Y=1$ under the action of $m$ explanatory variables. On the basis of the above model settings, when estimating the optimal adjustment parameters of the logistic source term, it is also necessary to add a penalty term to the 


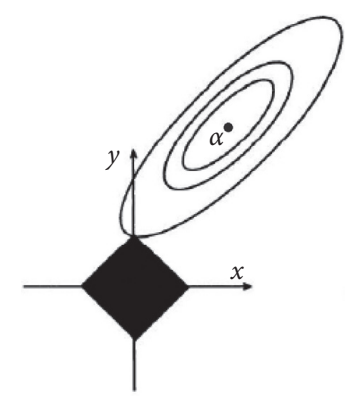

Figure 1: Regression model of logistic source term after logit transformation.

parameters to realize the estimation of parameters and the selection of variables [10].

Suppose the response variable $Y$ is a binary variable, whose values are $Y=1$ and $Y=0$. The $m$ explanatory variables that affect the value of $Y$ are $X_{1}, X_{2}, \ldots, X_{m}$. Suppose that there are $n$ observations in the data set; that is, there are $\left(x_{1}, y_{1}\right), \ldots,\left(x_{k}, y_{k}\right), \ldots,\left(x_{n}, y_{n}\right)$, where the observation $x_{k}=\left(x_{k 1}, x_{k 2}, \ldots, x_{k c}\right)$ is a $p$-dimensional vector, $y_{k} \in\{0,1\}$, and $k=1,2, \ldots, n$. Then, the following regression model of logistic source term is considered in the following equation:

$$
\ln \left(\frac{P_{k}^{\prime}}{1-P_{k}^{\prime}}\right)=\alpha_{0}+\alpha_{1} x_{k 1}+\alpha_{2} x_{k 2}+\cdots+\alpha_{p} x_{k p}
$$

where $P_{k}^{\prime}=P\left(y_{k}=1 \mid x_{k}\right)$ is the conditional probability of the result $y_{k}=1$ under the given $x_{k}$ condition. Similarly, the conditional probability of $y_{k}=0$ is $P\left(y_{k}=0 \mid x_{k}\right)=1-P^{\prime}$. Combining (8), the probability of an observation value is obtained as follows:

$$
P\left(y_{k}\right)=O P_{k}^{\prime} y_{k}\left(1-P^{\prime}\right)^{1-y_{k}}
$$

It is known that observations are independent of each other, so their joint distribution can be expressed as the product of each marginal distribution; then the likelihood function of $n$ observations is as follows:

$$
F(\alpha)=\prod_{k=1}^{n} P_{k}^{\prime} y_{k}\left(1-P^{\prime}\right)^{\left(1-y_{k}\right)} \text {. }
$$

In the above equation, $F(\alpha)$ represents the likelihood function for all events $\alpha$. According to the above equation, the log likelihood function is obtained as follows:

$$
\begin{aligned}
& \ln (F(\alpha))=\ln \left(\prod_{k=1}^{n} P_{k}^{\prime} y_{k}\left(1-P^{\prime}\right)^{\left(1-y_{k}\right)}\right)=\sum_{k=1}^{n}\left[y_{k} \ln P_{k}^{\prime}+\left(1-y_{k}\right) \ln \left(1-P^{\prime}\right)\right] \\
& =\sum_{k=1}^{n}\left[y_{k} \ln \left(\frac{P_{k}^{\prime}}{1-P_{k}^{\prime}}\right)+\ln \left(1-P^{\prime}\right)\right] \\
& =\sum_{k=1}^{n}\left[y_{k}\left(\alpha_{0}+\sum_{k^{\prime}=1}^{P^{\prime}} \alpha_{k^{\prime}} x_{k k^{\prime}}\right)-\ln \left(1+\exp \left(\alpha_{0}+\sum_{k^{\prime}=1}^{P^{\prime}} \alpha_{k^{\prime}} x_{k k^{\prime}}\right)\right)\right] \text {. } \\
& f_{\lambda}(\alpha)=-s(\alpha)+\lambda \sum_{k=1}^{p}\left|\alpha_{k}\right|
\end{aligned}
$$

$\alpha_{p} x_{k p} . k^{\prime}$ represents the observation value adjacent to $k$ observation value; $\alpha_{k^{\prime}}$ represents the event corresponding to adjacent observation value; $x_{k k^{\prime}}$ represents the relationship parameter between two adjacent observation values. According to the related theory of Lasso method, the coefficient estimates $\alpha^{\prime}$ in Lasso-logistic regression model are given by the minimum value of convex function of the following equation:
In the equation, $S(\alpha)$ is the logarithmic likelihood function of the estimated value $\alpha^{\prime}$. The coefficient estimates $\alpha^{\prime}$ can be written as follows:

$$
\alpha^{\prime}=\underset{\alpha}{\arg \min }\left[\sum_{k=1}^{n}\left(y_{k}\left(\alpha_{0}+\sum_{k^{\prime}=1}^{p} \alpha_{k^{\prime}} x_{k k^{\prime}}\right)-\ln \left(1+\exp \left(\alpha_{0}+\sum_{k^{\prime}=1}^{p} \alpha_{k^{\prime}} x_{k k^{\prime}}\right)\right)\right)+\lambda \sum_{k=1}^{p}\left|\alpha_{k}\right|\right] .
$$

The above equation can be divided into two parts: in the first part, $\quad \sum_{k=1}^{n}\left(y_{k}\left(\alpha_{0}+\sum_{k^{\prime}=1}^{p} \alpha_{k^{\prime}} x_{k k^{\prime}}\right)-\ln (1+\exp \right.$ $\left.\left.\left(\alpha_{0}+\sum_{k^{\prime}=1}^{p} \alpha_{k^{\prime}} x_{k k^{\prime}}\right)\right)\right)$ represents the fitting degree of the model; in the second part, $\lambda \sum_{k=1}^{p}\left|\alpha_{k}\right|$ represents the punishment degree for the selected variables, where $\lambda$ represents the adjustment parameter, and there is $\lambda \geq 0$. Finally, the selected variable set should minimize the values of these two parts [11]. 
Using Lasso-logistic model for variable selection, the key is to adjust the parameter $\lambda$. The size of $\lambda$ affects whether the coefficient of some variables can be compressed to 0 in the process of variable selection, which will directly affect the result of variable selection. $K$-fold cross validation, generalized cross validation, and BIC criterion are also suitable for Lasso-logistic model. In this analysis, $K$-fold cross validation is used to select the adjustment parameters.

Let $L(y, g(x))$ be the loss function. Firstly, the data set is randomly divided into $K$ parts with approximately the same capacity. The first $k(k=1,2, \ldots, K)_{\text {-th }}$ data is taken out as the verification set in turn, and the remaining $K-1_{- \text {th }}$ data is taken as the training set. Finally, the verification results on all $K$ data are combined to obtain the $\mathrm{CV}$ value of $K$-fold cross validation; namely, the pretest error of $K$-fold cross validation is shown in the following equation:

$$
\mathrm{CV}=\frac{1}{n} \sum_{k=1}^{n} L\left(y_{k}, \widehat{g}_{-k(k)}\left(x_{k}\right)\right) \text {, }
$$

where $n$ is the sample size, $y_{k}$ is the observation value of the $k_{\text {-th }}$ sample, $k(k)$ is the number of the $k_{\text {-th }}$ sample, and $g_{-k(k)}$ is the model fitted on the remaining $K-1_{- \text {th }}$ data after removing the $k(k)_{- \text {th }}$ data. It is assumed that a group of models are fitted and expressed as $\widehat{g}_{-k}(x, \lambda)$, and the model contains the adjustment parameter $\lambda$ [12]. Then the prediction error obtained by $K$-fold cross validation is shown in the following equation:

$$
\mathrm{CV}(\lambda)=\frac{1}{n} \sum_{k=1}^{n} L\left(y_{k}, \widehat{g}_{-k}(x, \lambda)\right) .
$$

Finding $\lambda$, which makes $\operatorname{CV}(\lambda)$ the minimum, is the optimal adjustment parameter of Lasso-logistic model, so as to reset the optimal adjustment parameter of logistic source term.

2.2. Quantitative Analysis of the Global Existence and Solutions' Limitations of Chemotaxis Equations with Logistic Source Terms. In order to analyze the solution of chemotaxis equations with logistic source term, it is necessary to obtain the global existence and limitation of the solution of chemotaxis equations by quantitative analysis. The known chemotaxis equations with logistic source term are as follows:

$$
\left\{\begin{array}{l}
x_{t}=\Delta x-\nabla(x \Delta x)+\mu x-\sigma x^{2}, \quad x \in \Omega, t>0, \\
z_{t}=\Delta z-x z, \quad x \in \Omega, t>0, \\
\frac{\partial x}{\partial n}=\frac{\partial z}{\partial n}=0, \quad(u, t) \in \partial \Omega \times(0, T), \\
x(u, 0)=x_{0}(u), \\
z(u, 0)=z_{0}(u), \quad x \in \Omega .
\end{array}\right.
$$

In the above equations, $\Omega \subset *^{3}$ is the bounded region with smooth boundary, $n$ is the normal vector outside the unit on $\partial \Omega, \mu \in *, \sigma>0 . x_{t}$ represents the trend primary equation under the influence of time series parameters; $z_{t}$ represents the dependent variable affected by the result of the equation; $\Delta x$ and $\Delta z$ represent the difference value, respectively; $T$ represents the periodic parameter; $u$ represents the variable of the trend equation. The global existence and boundedness of initial boundary value solutions are discussed [13]. Assume that the initial value meets the conditions shown in the following equation:

$$
\left\{\begin{array}{l}
x_{0} \in Q^{0}(\bar{\Omega}), \quad x_{0} \geq 0, u \in \bar{\Omega}, \\
z_{0} \in W^{(1, \infty)}(\Omega), \quad z_{0} \geq 0, u \in \bar{\Omega} .
\end{array}\right.
$$

The classical solutions of homogeneous initial boundary value problems for chemotactic systems are global and bounded. If the above conditions are true, then when the adaptive parameter $\sigma \geq 3+(23 / 3)\left\|z_{0}\right\|_{L^{\infty}(\Omega)}^{2}$ is limited, the system of (19) is in the only bounded global classical solution $(x, z)$, and there are constants at the same time. For all the time series parameters $Q>0$, there are

$$
\|x(\cdot, t)\|_{L^{\infty}(\Omega)}+\|z(\cdot, t)\|_{W^{(1, \infty)}(\Omega)} \leq Q .
$$

Among the above equations, $L$ represents the constraint function of missing equation and $W$ represents the maintenance function. When the constant $Q$ satisfies the above equation, the assumption is true; that is, the initial value assumption is true, which can quantitatively analyze the global existence and limitations of the solution of the chemotactic equation system [14]. It is known that, for any conditional parameter $t \in\left(0, T_{\max }\right)$, there is a condition shown in the following equation:

$$
\frac{\mathrm{d}}{\mathrm{d} t} \int_{\Omega} x^{2}+\int_{\Omega}|\nabla x|^{2} \leq \int_{\Omega} x^{2}|\nabla x|^{2}+2 \mu \int_{\Omega} x^{2}-2 \sigma \int_{\Omega} x^{3} .
$$

It is proved that in (19), both sides of the first equation are multiplied by $x$ at the same time and both sides are integrated. For any $t \in\left(0, T_{\max }\right)$, the following equation can be obtained:

$$
\frac{1}{2} \frac{\mathrm{d}}{\mathrm{d} t} \int_{\Omega} x^{2}=-\int_{\Omega}|\nabla x|^{2}+\int_{\Omega} x \nabla x \cdot \nabla x+\int_{\Omega} x^{2}-\sigma \int_{\Omega} x^{3} .
$$

Because of Young inequality, we can get $\int_{\Omega} x \nabla x \cdot \nabla z \leq(1 / 2) \int_{\Omega}|\nabla x|^{2}+(1 / 2) \int_{\Omega} x^{2}|\nabla z|^{2}$. Therefore, for any $t \in\left(0, T_{\max }\right)$, there are

$$
\begin{aligned}
\frac{\mathrm{d}}{\mathrm{d} t} \int_{\Omega}|\nabla z|^{4}+\left.\left.\int_{\Omega}|\nabla| \nabla z\right|^{2}\right|^{2} \leq & 7\left\|z_{0}\right\|_{L^{\infty}(\Omega)}^{2} \int_{\Omega} x^{2}|\nabla z|^{2} \\
& +2 \int_{\partial \Omega}|\nabla z|^{2} \frac{\partial|\nabla z|^{2}}{\partial n} .
\end{aligned}
$$

According to the known conditions, the existence analysis in (25) is also obtained by combining Young's inequality. 


$$
\begin{aligned}
& \frac{\mathrm{d}}{\mathrm{d} t}\left[4 \int_{\Omega} x^{2}+\int_{\Omega}|\nabla z|^{2}+\int_{\Omega} x|\nabla z|^{2}\right]+\int_{\Omega}|\nabla x|^{2}+\left.\left.\frac{1}{12} \int_{\Omega}|\nabla| \nabla z\right|^{2}\right|^{2} 8 \sigma \int_{\Omega} x^{3} \\
& \quad+\left(\sigma-3-\frac{23}{3}\left\|z_{0}\right\|_{L^{\infty}(\Omega)}^{2}\right) \int_{\Omega} x^{2}|\nabla z|^{2} \\
& \quad \leq 8 \mu \int_{\Omega} x^{2}+\mu \int_{\Omega} x|\nabla z|^{2}+2 \int_{\partial \Omega}|\nabla z|^{2} \frac{\partial|\nabla z|^{2}}{\partial n}+2 \int_{\partial \Omega} x \frac{\partial|\nabla z|^{2}}{\partial n} .
\end{aligned}
$$

According to (24), the above conclusions are obtained. When the boundary constraint parameter $\mu$ and separation parameter $\sigma$ meet (25), it is proved that the solution of the chemotactic equation system is complete, so that the general curve of the chemotactic equation can be obtained [15]. Figures 2(a) and 2(b) show the general curve of chemotaxis equations.

According to the curve trend in the figure above, under the influence of two parameters, the result curve obtained by solving has symmetry characteristics, so it can be seen that the whole solution of chemotaxis equation system with logistic source term has complete existence [16]. Next, we will prove the global limitation of the solution.
If $\sigma \geq 3+(23 / 3)\left\|z_{0}\right\|_{L^{\infty}(\Omega)}^{2}$ is still assumed, then there is a constant $Q^{\prime}$ for any $t \in\left(0, T_{\max }\right)$, which makes the following equations set up:

$$
\left\{\begin{array}{l}
\int_{\Omega} x^{2}(\cdot, t) \leq Q^{\prime}, \\
\int_{\Omega}|\nabla z(\cdot, t)|^{4} \leq Q^{\prime} .
\end{array}\right.
$$

Then, let $h(t)=4 \int_{\Omega} x^{2}+\int_{\Omega}|\nabla z|^{4}+\int_{\Omega} x|\nabla z|^{2}$; the quantitative analysis process exists in the same reason, and the inequality can be gotten:

$$
\begin{aligned}
& h^{\prime}(t)+h(t)+\int_{\Omega}|\nabla x|^{2}+\left.\left.\frac{1}{12} \int_{\Omega}|\nabla| \nabla z\right|^{2}\right|^{2}+8 \sigma \int_{\Omega} x^{3} \\
& \quad \leq(8 \mu+4) \int_{\Omega} x^{2}+(\mu+1) \int_{\Omega} x|\nabla z|^{2}+\int_{\Omega}|\nabla z|^{4}+2 \int_{\partial \Omega}|\nabla z|^{2} \frac{\partial|\nabla z|^{2}}{\partial n}+\int_{\partial \Omega} x \frac{\partial|\nabla z|^{2}}{\partial n} .
\end{aligned}
$$

Using Young's inequality for (27), we can get that, for any $\mu>0, \sigma>0$, there is a constant $\widehat{Q}>0$. It is known that $\int_{\Omega}|\nabla z|^{2} \leq U$, where $U$ is the effective constant; combining
(18), then according to Gagliardo-Nirenberg interpolation inequality, we can get

$$
\begin{gathered}
\|\nabla z(\cdot, t)\|_{L^{\infty}(\Omega)} \leq\left\|\nabla e^{t \Delta} z(\cdot, 0)\right\|_{L^{\infty}(\Omega)}+\int_{0}^{t}\left\|\nabla e^{(t-s) \Delta} x(\cdot, s) z(\cdot, s)\right\|_{L^{\infty}(\Omega)} \\
\leq Q_{1}+\int_{0}^{t} Q_{2}\left(1+(t-s)^{-(1 / 2)}\right) e^{-\lambda_{1}(t-s)}\left\|\nabla e^{(t-s) \Delta} x(\cdot, s) z(\cdot, s)\right\|_{L^{\infty}(\Omega)}
\end{gathered}
$$

In the above equation, $e$ represents the natural base number; $s$ represents the failure sequence. $\lambda_{1}>0$, which is the first nonzero eigenvalue of the operator $\nabla$ in the bounded region $\Omega$ under the boundary condition. Because $\|x(\cdot, s)\|_{L^{\infty}(\Omega)}$ has been proved, it can judge the solution of chemotaxis equations with logistic source term, which has limitations [17].

2.3. Evaluating the Well-Posed Behavior of Solutions of Chemotaxis Equations. On the basis of the above analysis, the global existence and limitations of the solutions of the chemotaxis equations are determined to evaluate the wellposed behavior of the solutions of the chemotaxis equations. According to the limitation of the solution of the chemotactic equation system for logistic source term, the linear term of the equation system is controlled. In this paper, the symmetrizer chemotaxis equation is used to simplify and form a symmetric hyperbolic function compatible with the boundary [18]. However, the difficulty in this process lies in how to select proper symmetrizer to make some linear terms disappear in the process of symmetrization evaluation. On the other hand, after choosing the proper symmetrizer and transforming the equation with logistic source term into the chemotaxis equation with symmetric hyperbolic rate, due to the particularity of the boundary, it cannot directly use the energy estimation to get the estimation of the spatial derivative and the spatialtemporal mixed derivative but needs to use the time derivative to control and then gets their estimates. The relevant 


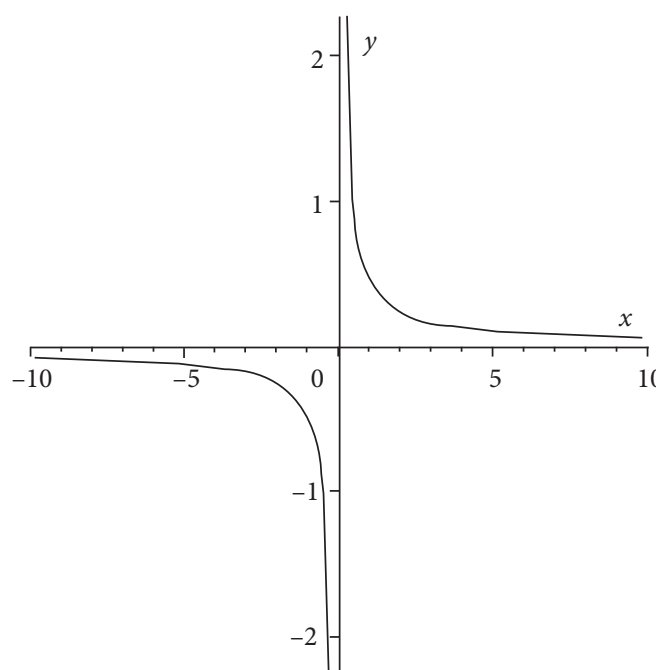

(a)

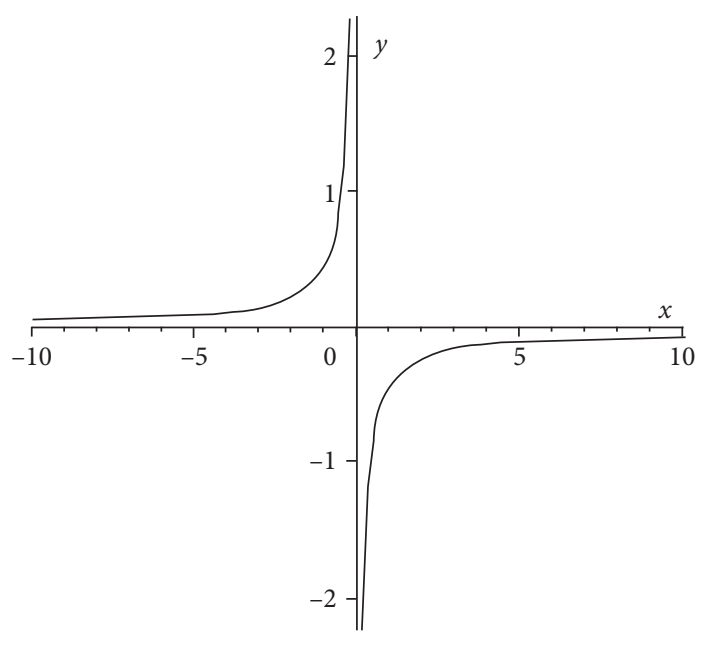

(b)

Figure 2: General curve of chemotaxis equation when $\mu>0, \sigma>0$ and when $\mu<0, \sigma<0$. (a) Parameter branch graph when $\mu>0, \sigma>0$. (b) Parameter branch graph when $\mu<0, \sigma<0$.

chemotaxis equation is given again, and the well-posed solution of the chemotaxis equation group with logistic source term is evaluated. The equation is the same as (19), and the quasi-transformed steady-state equation group is as follows:

$$
\left\{\begin{array}{l}
\nabla \varepsilon_{1}=\varepsilon_{1} \nabla \Theta, \\
\nabla \varepsilon_{2}=-\varepsilon_{2} \nabla \Theta, \\
\Delta \Theta=\varepsilon_{1}-\varepsilon_{2}-F(x) .
\end{array}\right.
$$

In the equation, $\varepsilon_{1}$ and $\varepsilon_{2}$ represent the intrinsic parameter and the well-posed parameter, respectively; $\nabla \varepsilon_{1}$ and $\nabla \varepsilon_{2}$ represent the gradient operator of the two parameters, respectively; $\Theta$ represents the behavior condition; $\nabla \Theta$ represents the gradient operator of behavior condition; $\Delta \Theta$ represents the missing function operator of the behavior condition; $F(x)$ represents the steady-state control function. The specific description equation of behavior conditions is as follows:

$$
\left.\nabla \Theta \cdot \kappa\right|_{\partial \Omega}=0
$$

In the equation, $\kappa$ represents the set of behavior condition constraints. In order to meet the practical application, it is necessary to consider that the solution of chemotaxis equation system meets the stability requirements, so as to ensure that the solution result will not be completely unbalanced due to the change of a certain parameter. The satisfied condition is $\inf _{x} \varepsilon_{1}>0, \inf _{x} \varepsilon_{2}>0$.

To solve the ordinary differential equation, combined with (29), there is a new equation:

$$
\nabla \Theta=\frac{\nabla \varepsilon_{1}}{\varepsilon_{1}}=\nabla\left(\ln \varepsilon_{1}\right)=-\frac{\nabla \varepsilon_{2}}{\varepsilon_{2}}=-\nabla\left(\ln \varepsilon_{2}\right) .
$$

According to the satisfied conditions, the above equations are solved and the following system of equation is obtained:

$$
\left\{\begin{array}{l}
\varepsilon_{1}(x)=Q_{1} e^{\Theta} \\
\varepsilon_{2}(x)=Q_{1} e^{-\Theta}
\end{array}\right.
$$

In the above equations, $Q_{1}$ is an unknown normal number. Taking (32) into the third equation and (3)-(2) of (29), combining with boundary conditions, the following elliptic equation can be obtained:

$$
\left\{\begin{array}{l}
\Delta \Theta=Q_{1} e^{\Theta}-Q_{1} e^{-\Theta}-F(x), \text { in }(\Omega), \\
\frac{\partial \Theta}{\partial \kappa}=0, \text { on }(\partial \Omega) .
\end{array}\right.
$$

Therefore, if we want to obtain the well-posed solutions of the steady-state equations (29) with insulation boundary conditions, that is, (30), it is equivalent to getting the wellposed solutions of the elliptic equations (33) [19]. According to the elliptic equations, the corresponding variational functional can be taken as in the following equation:

$$
I(\Theta)=\int_{\Omega} \frac{|\nabla \Theta|^{2}}{2}+Q_{1} e^{\Theta}+Q_{1} e^{-\Theta}-F(x) \Theta \mathrm{d} x .
$$

According to the above equation, the general curve of the elliptic system of equations is drawn. According to the maximum and minimum values of the curve changes, the extremum element of the functional is found out; that is, the function that makes this functional takes the extremum; namely, the solution of the elliptic system of equations [20]. Figure 3 is the curve diagram of the elliptic equation.

According to the intersection points marked in the above figure, it proves the minimizer of functional $I$ in space $D=\left\{\Theta \mid\left(\int_{\Omega}|\nabla \Theta|^{2} \mathrm{~d} x\right)^{1 / 2}+\left(\int_{\Omega}|\Theta|^{2} \mathrm{~d} x\right)^{1 / 2}<\infty, x \in \Omega\right\}$. The norms defining the space $D$ are

$$
\|\Theta\|_{D}=\left(\int_{\Omega}|\nabla \Theta|^{2} \mathrm{~d} x\right)^{1 / 2}+\left(\int_{\Omega}|\Theta|^{2} \mathrm{~d} x\right)^{1 / 2} .
$$




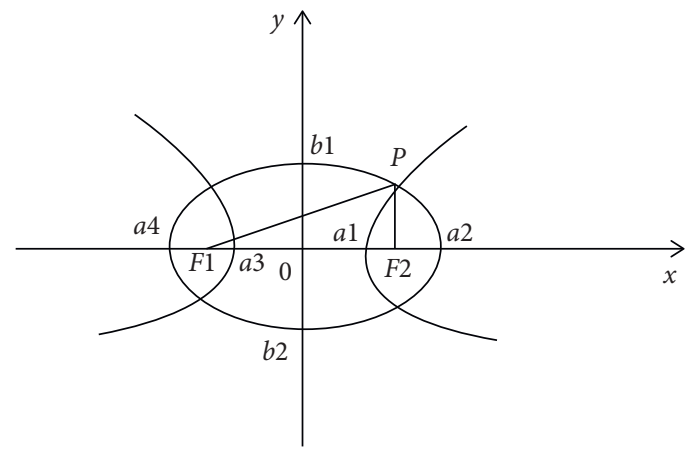

Figure 3: Schematic diagram of the elliptic equation curve.

TABLE 1: Sensitivity and specificity parameters that affect chemotaxis equations.

\begin{tabular}{lcc}
\hline Test group & Sensitivity & Specificity \\
\hline T1 & 0.60 & 0.60 \\
T2 & 0.74 & 0.48 \\
T3 & 0.97 & 0.27 \\
T4 & 0.52 & 0.58 \\
T5 & 0.57 & 0.73 \\
T6 & 0.91 & 0.29 \\
T7 & 0.68 & 0.64 \\
T8 & 0.87 & 0.43 \\
T9 & 0.96 & 0.25 \\
T10 & 0.61 & 0.76 \\
T11 & 0.77 & 0.66 \\
T12 & 0.96 & 0.39 \\
T13 & 0.61 & 0.63 \\
T14 & 0.70 & 0.59 \\
T15 & 0.95 & 0.22 \\
T16 & 0.27 & 0.75 \\
T17 & 0.47 & 0.80 \\
T18 & 0.92 & 0.31 \\
T20 & 0.41 & 0.65 \\
\hline
\end{tabular}

If we can find the minimizer of functional $I$ in space $D$, it can say that (33) have solutions in space D. Obviously, (34) has lower bounds. Let $v=\inf _{D} I(\Theta)$, from the definition of the lower bound, we can see that there is a minimization sequence $\{\Theta\} \in D(\Omega)$, which makes the equation $\lim _{n \rightarrow \infty} I\left(\Theta_{n}\right)=v$ hold. It can be seen from observation and calculation that the variational functional is in the following equation:

$$
I\left(\Theta_{n}\right)=\int_{\Omega} \frac{\left|\nabla \Theta_{n}\right|^{2}}{2}+Q_{1} e_{n}^{\Theta}+Q_{1} e^{-\Theta_{n}}-F(x) \Theta_{n} \mathrm{~d} x .
$$

$I\left(\Theta_{n}\right)$ of the equation is a convex functional. In the next step, we need to know whether $\left\|\Theta_{n}\right\|_{D} \leq Q_{1}$ holds, where $Q_{1}$ represents a positive constant. By using the counterproof method, supposing that there is $\left\|\Theta_{n}\right\|_{D} \longrightarrow \infty$, whether $\Theta_{n}$ is positive or negative, there is $e^{\Theta_{n}}>\Theta_{n}^{2}$, so there will be $I\left(\Theta_{n}\right) \longrightarrow \infty$. But this contradicts the fact that $\left\{\Theta_{n}\right\}$ is a minimization sequence of $I$, so $\left\|\Theta_{n}\right\|_{D} \leq Q_{1}$ holds. According to this equation, there is a weak convergence subsequence $\left\{\Theta_{n i}\right\}$ of the minimization sequence $\left\{\Theta_{n}\right\}$, so that when $n i \longrightarrow \infty$, there is a limit $\Theta$ in $D(\Omega)$ that satisfies $\Theta_{n i} \longrightarrow \Theta$, so that the evaluation on the well-posed behavior of the solution of the chemokine equation system is completed, and the analysis of the solution of the chemokine equation system with logistic source term is realized [21].

\section{Test Results and Analysis}

Compared with the traditional analysis method, the two analysis methods consider the calculation error when dealing with the solution of the chemotaxis equations. The test results of the proposed analysis method are set as experiment $A$ and the test results of the traditional analysis method as experiment $B$. The sensitivity index and specificity index of the parameters of the experimental test object are set, and two analysis methods are used to analyze the solution of the chemotaxis equations with sensitivity and specificity parameters. Table 1 shows the sensitivity and specificity values.

Two analysis methods are used to analyze the solution of chemotaxis equations with logistic source term under the influence of sensitivity and specificity parameters, and the 


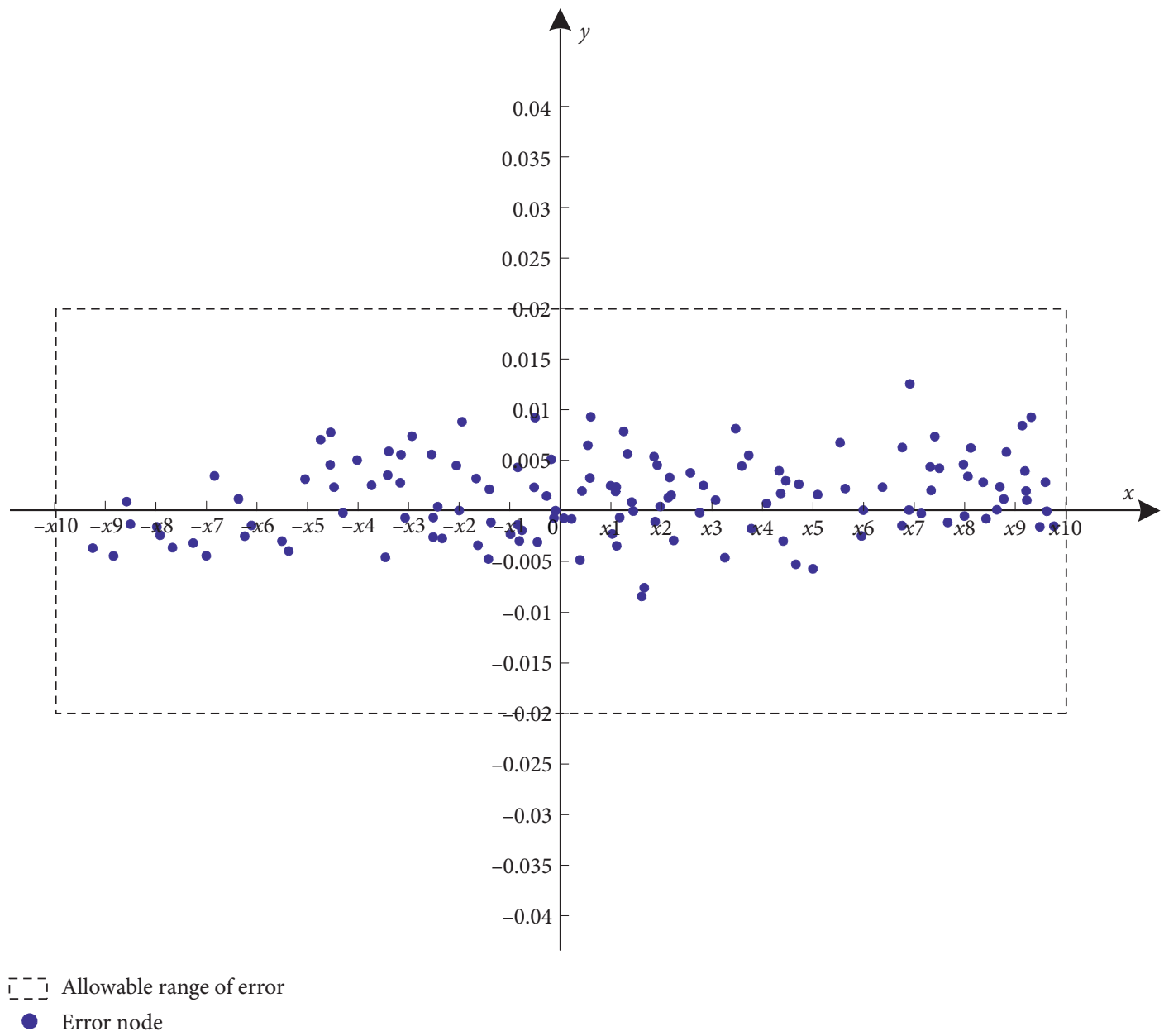

FIgURE 4: Test results of the experimental group A under the proposed method.

calculation errors are compared under the influence of two groups of data. The reliability evaluation model and data error prediction model are established to identify whether the error value of the two analysis methods is within the allowable range. The calculation equation of the reliability model is as follows:

$$
R(x)=1-\lambda_{(a, b)}(S, C) .
$$

In the equation, $R(x)$ is the reliability measurement result of the analysis result $x ; \lambda_{(a, b)}$ is the allowable variation of the parameter within the allowable error range $(a, b) ; S$ is the support parameter; $C$ is the reliability parameter. At the same time, the error prediction model is as follows:

$$
E P(x)=\omega R(x)+\frac{1}{\beta} \sum_{i=1}^{n} k \mu_{i} .
$$

In the equation, $\omega$ is the prediction weight coefficient; $\beta$ is the prediction limit parameter; $n$ is the number of direct predictions; $k$ is the linear parameter; $\mu_{i}$ is the floating value of the $i_{- \text {th }}$ group of test data. Figures 4 and 5 are the analysis error diagrams of the two methods when the sensitivity and specificity parameters are different.

It is known that, in the above two groups of test results, the dotted box represents the allowable error range, and the solid dot represents the error node. According to the distribution of error nodes, we can see that, under the proposed analysis method, the error nodes in group $A$ are completely included in the allowable range of error, these errors are relatively concentrated, and the difference floating is very small. However, under the traditional analysis method, some of the error nodes in group $B$ exceed the allowable error range, and the error nodes in the range are relatively scattered with different values. According to the test results 


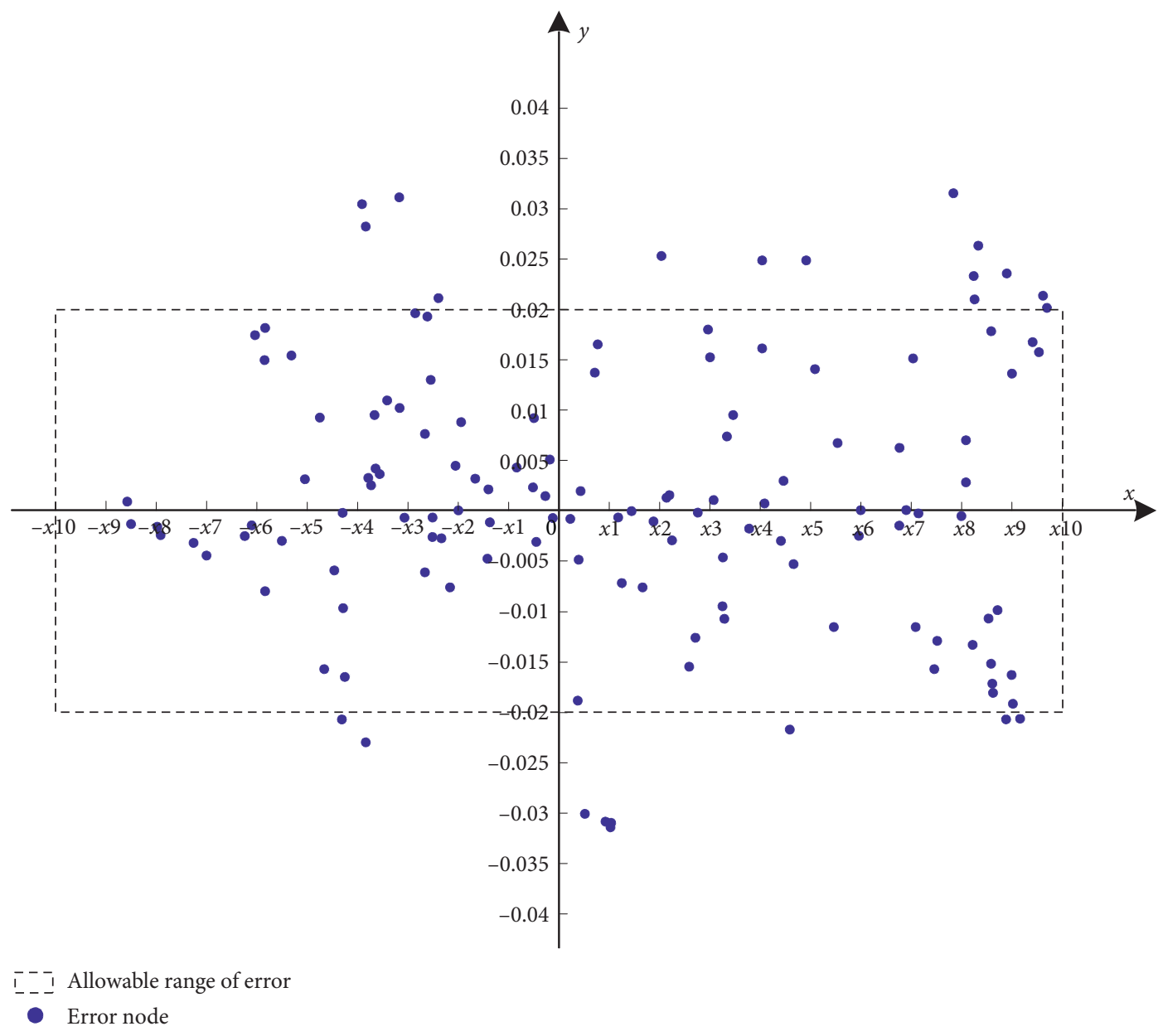

Figure 5: Experimental group B test results under traditional analysis methods.

of the two groups, the proposed analysis method is superior to the traditional analysis method in controlling the calculation error.

\section{Conclusions}

According to the solution, problem of chemotaxis equations with logistic source term, a more targeted analysis method is proposed. The sensitivity index and specificity index of the parameters of the experimental test object were set, and two analysis methods were used to analyze the solution of the chemotaxis equations with sensitivity and specificity parameters. Through quantitative analysis, the global existence and limitations of the solution of chemotaxis equations are obtained. The reliable control of the calculation error is realized. Accuracy of the solution result is improved, and a more cautious analysis method is provided for the solution problem of equations in the mathematical field. It was found that the proposed analysis method is better when compared to the traditional analysis method in controlling the calculation error.

\section{Data Availability}

The data used to support the findings of this study are available upon request.

\section{Conflicts of Interest}

The authors declare that there are no conflicts of interest regarding the publication of this paper.

\section{References}

[1] M.-F. Danca, M. Fečkan, and N. V. Kuznetsov, "Chaos control in the fractional order logistic map via impulses," Nonlinear Dynamics, vol. 98, no. 2, pp. 1219-1230, 2019.

[2] E. F. Keller and L. A. Segel, "Model for chemotaxis," Journal of Theoretical Biology, vol. 30, no. 2, pp. 225-234, 1971.

[3] M. Rakhra, R. Singh, T. K. Lohani, and M. Shabaz, "Metaheuristic and machine learning-based smart engine for renting and sharing of agriculture equipment," Mathematical Problems in Engineering, vol. 2021, Article ID 5561065, 13 pages, 2021.

[4] V. Jagota, A. P. S. Sethi, and K. Kumar, "Finite element method: an overview," Walailak Journal of Science \& Technology, vol. 10, no. Issue 1, pp. 1-8, 2013.

[5] N. Taneja, M. Shabaz, and V. Khajuria, "Iris detection using segmentation techniques," International Journal of Computer Sciences and Engineering, vol. 6, no. 9, pp. 442-444, 2018.

[6] J. L. Fleiss, J. B. S. Williams, and A. F. Dubro, "The logistic regression analysis of psychiatric data," Journal of Psychiatric Research, vol. 20, no. 3, pp. 145-209, 1986. 
[7] D. Singh, A. Chhabra, and J. Singh, "IMCLA: performance evaluation of integrated multilevel checkpointing algorithms using checkpointing efficiency," International Journal of Computing and Digital Systems, vol. 2, no. 1, pp. 9-19, 2013.

[8] G. Brightwell, T. House, and M. Luczak, "Extinction times in the subcritical stochastic SIS logistic epidemic," Journal of Mathematical Biology, vol. 77, no. 2, pp. 455-493, 2018.

[9] J. Yao and D. Liu, "Logistic regression analysis of risk factors for intracranial infection after multiple traumatic craniotomy and preventive measures," Journal of Craniofacial Surgery, vol. 30, no. 7, pp. 1946-1948, 2019.

[10] G. Reibnegger, D. Fuchs, A. Hausen et al., "Generalized likelihood ratio concept and logistic regression analysis for multiple diagnostic categories," Clinical Chemistry, vol. 35, no. 6, pp. 990-994, 2019.

[11] R. W. Veltri, M. Chaudhari, M. C. Miller et al., "Comparison of logistic regression and neural net modeling for prediction of prostate cancer pathologic stage," Clinical Chemistry, vol. 48, no. 10, pp. 1828-1834, 2020.

[12] S. A. Parah, N. A. Loan, A. A. Shah et al., "A new secure and robust watermarking technique based on logistic map and modification of DC coefficient," Nonlinear Dynamics, vol. 93, no. 7, pp. 1933-1951, 2018.

[13] Y. N. Alvarez and R. Sa Earp, "Sharp solvability criteria for Dirichlet problems of mean curvature type in Riemannian manifolds: non-existence results," Calculus of Variations and Partial Differential Equations, vol. 58, no. 6, pp. 197-211, 2019.

[14] B. H. Hwang and S. B. Yun, "Anderson-witting model of the relativistic Boltzmann equation near equilibrium," Journal of Statistical Physics, vol. 176, no. 12, pp. 1009-1045, 2019.

[15] D. Bonheure, J.-B. Castéras, T. Gou, and L. Jeanjean, "Normalized solutions to the mixed dispersion nonlinear Schrödinger equation in the mass critical and supercritical regime," Transactions of the American Mathematical Society, vol. 372, no. 3, pp. 2167-2212, 2019.

[16] J. E. Elkhoury, R. Shankar, and T. S. Ramakrishnan, "Resolution and limitations of X-Ray Micro-CT with applications to sandstones and limestones," Transport in Porous Media, vol. 129, no. 7, pp. 1-13, 2019.

[17] Y. K. Tovbin, "Small systems and limitations on the use of chemical thermodynamics," Russian Journal of Physical Chemistry A, vol. 92, no. 1, pp. 1-18, 2018.

[18] A. Alberico, I. Chlebicka, A. Cianchi, and A. ZatorskaGoldstein, "Fully anisotropic elliptic problems with minimally integrable data," Calculus of Variations and Partial Differential Equations, vol. 58, no. 6, p. 186, 2019.

[19] D. Bolin, K. Kirchner, and M. Kovács, "Weak convergence of Galerkin approximations for fractional elliptic stochastic PDEs with spatial white noise," Bit Numerical Mathematics, vol. 58, no. 4, pp. 881-906, 2018.

[20] I. Rácz, "On the evolutionary form of the constraints in electrodynamics," Symmetry, vol. 11, no. 1, p. 10, 2018.

[21] X. He, G. Liu, B. Li et al., "Erratum: functional analysis of the heterotrimeric NF-Y transcription factor complex in cassava disease resistance," Annals of Botany, vol. 124, no. 7, p. 1243, 2019. 